\title{
Study of prescription pattern of antimicrobials used in post-operative surgical patients in tertiary care hospital
}

\author{
Jitendra H. Hotwani, Nishikant H. Madkholkar*
}

Department of Pharmacology, Topiwala National Medical College, Mumbai, Maharashtra, India

\author{
Received: 17 March 2021 \\ Accepted: 12 April 2021 \\ *Correspondence: \\ Dr. Nishikant H. Madkholkar, \\ Email: madkholkarnishikant@gmail.com
}

Copyright: (C) the author(s), publisher and licensee Medip Academy. This is an open-access article distributed under the terms of the Creative Commons Attribution Non-Commercial License, which permits unrestricted non-commercial use, distribution, and reproduction in any medium, provided the original work is properly cited.

\begin{abstract}
Background: Antimicrobials are used for prophylaxis and treatment of infections which occur following surgical procedures, to reduce the morbidity and mortality associated with surgical site infections (SSIs). A surgical site infection is an infection that occurs after surgery in the part of the body where the surgery took place. These are infections of the tissues, organs, or spaces exposed by surgeons during performance of an invasive procedure.

Methods: A prospective, non-interventional, observational study in tertiary care hospital for duration of 9 months. Sample size was 330 .

Results: The prescription pattern shows that nitroimidazoles were the most commonly prescribed group of antimicrobials in $72 \%$ of patients followed by penicillins $(58 \%)$ and cephalosporins (42\%). Metronidazole (72\%), amoxicillin and clavulanic acid $(51.21 \%)$ ceftriaxone $(20.90 \%)$ were most commonly prescribed antimicrobials in these groups. Two antimicrobials were prescribed in $47 \%$ patients with nitroimidazole and penicillins being the most commonly prescribed combination of antimicrobial. Three antimicrobials were prescribed in $25 \%$ patients and four antimicrobials in $8 \%$ patients. This shows trend towards polypharmacy. About $82 \%$ of antimicrobials were prescribed by brand names and $64 \%$ of total antimicrobials prescribed from outside the hospital pharmacy source. About $12.42 \%$ of patients changed antimicrobial therapy after culture and sensitivity report.

Conclusions: Total duration, number of anti-microbial used was more in clean-contaminated, contaminated, dirty wound surgeries as compare to clean wound surgeries. Our study provides a framework for continuous prescription audit of antimicrobials in a hospital setting and thus can help in rational use of antimicrobials in post-operative surgical patients.
\end{abstract}

Keywords: Surgical site infections, Clean, Clean contaminated, Dirty wounds

\section{INTRODUCTION}

Antimicrobials are used for prophylaxis and treatment of infections which occur following surgical procedures, to reduce the morbidity and mortality associated with surgical site infections (SSIs). A surgical site infection is an infection that occurs after surgery in the part of the body where the surgery took place. ${ }^{1}$ While the global estimates of surgical site infection (SSI) have varied from $0.5 \%$ to $15 \%$, studies in India have consistently shown higher rates ranging from $23 \%$ to $38 \%{ }^{3}$ The incidence of SSI may be influenced by factors such as pre-operative care, the theatre environment, post-operative care and the type of surgery. ${ }^{2}$

The type of surgeries has been classified by National Research Council (NRC) in four classes: clean, cleancontaminated, contaminated and dirty. ${ }^{3}$

Clean surgeries are those in which surgical wounds that exhibit no infection or inflammation; operations not involving the entry of respiratory, digestive, genital or 
urinary tracts example- hernia repair, hydrocele, breast lump excision etc. ${ }^{3}$

Clean-contaminated surgeries involve opening of the respiratory, digestive, genital or urinary tracts under controlled conditions and without abnormal contamination example- all elective surgeries such as cholecystectomy, appendicectomy, colorectal surgeries, small intestinal surgeries etc. ${ }^{3}$

Contaminated surgeries are fresh (within $7 \mathrm{~h}$ of causal event), include open trauma injuries. Wounds with acute, non-purulent inflammation are included in this class example penetrating abdominal trauma, large tissue injury, enterotomy during bowel obstruction etc. ${ }^{3}$

Dirty surgeries involve old (more than $7 \mathrm{~h}$ after causal event) trauma injuries with devitalised tissue and with preexisting clinical infection or perforated viscera example perforation of gastrointestinal, biliary or oropharyngeal tract before operation, necrotising soft tissue infections. ${ }^{3}$

Drug utilization study is defined as 'the marketing, distribution, prescription and use of drugs in society, with special emphasis on the resulting medical, social and economic consequences' and has the principle aim of facilitating the rational use of drugs. ${ }^{4}$

Antimicrobials used in treatment and prevention of surgical infections are beta-lactam antibiotics like penicillins, cephalosporins, aminoglycosides like gentamicin, tobramycin, quinolones like ciprofloxacin, ofloxacin, levofloxacin, imidazoles like metronidazole. ${ }^{5}$

Today antimicrobial drugs are one of the most commonly used drugs in clinical practice and the problems associated with their use is a global problem. In India, the prevalence of use of antimicrobials varies from 24-67\%. Irrational antimicrobials prescription leads to polypharmacy, increased expenses for drugs, preference of branded medicines over generic medicines, increased hospital stay and chances of developing resistance to antimicrobials. Hence, it is necessary to take several steps to improve the appropriate use of antibiotic. $^{6}$

Keeping these facts in consideration the present study was undertaken to analyse the current prescribing trends of postoperative antimicrobial therapy in surgical wards of a tertiary care hospital. We are also comparing the cost of antimicrobial therapy in different surgical classes.

\section{METHODS}

\section{Study design}

A prospective, non-interventional, observational study.

\section{Study site}

General Surgery Wards of a tertiary care hospital.

\section{Study duration}

9 months from the date of ECARP approval- 12 January 2018 to 12 October 2018 .

\section{Source of data}

Case papers and prescription of patients in surgery ward.

\section{Sample size}

Sample size was 330. Number of patients admitted in 4 surgery wards are approximately 65 per week under all six units. Therefore, in one month approximately 260 patients are admitted.

To represent 9-month post-operative surgical patients of different types of surgeries $(260 \times 9=2340)$, sample size calculated using $95 \%$ confidence level and $5 \%$ confidence interval as per sample size calculator is $330 .^{7}$

\section{Sampling technique}

Convenient sampling

\section{Patient selection}

\section{Inclusion criteria}

Patients undergone surgery and on post-operative antimicrobials therapy, Patients of either gender aged $\geq 18$ years.

\section{Exclusion criteria}

Patients of outpatient department in surgery.

\section{Study procedure}

Permission of Head of General Surgery \& Ethics committee for Academic Research Project (ECARP) was taken. Patients admitted in all 6 surgery units who were on post-operative antimicrobials treatment were enrolled in the study. Written informed consent was taken from patients or LAR (legally acceptable representative).

\section{Statistical analysis}

Descriptive statistical terms such as mean, standard deviation, median, percentage etc were used to describe the data which was collected.

For comparison of four surgical groups for parameters like duration of antimicrobial therapy, number of antimicrobials prescribed, cost of antimicrobial therapyKruskal Wallis test or one-way ANOVA followed by post hoc Tukey's test or Dunn's test as appropriate (SPSS version 23 was used for this). 


\section{RESULTS}

Total of 330 patients undergone surgical procedure in surgery department were enrolled during the study period of 9 months and they received total of 731 antimicrobials during their hospital stay. Mean age of the study population was $45.81 \pm 14.98$ years with range was between 18 and 85 years. Out of 330 patients percentage of male $(68.18 \%)$ was more than that of female $(31.82 \%) .141$ patients were having co-morbidities. Hypertension and diabetic mellitus were most common found comorbidity in $65(19.7 \%)$ and 51(15.4\%) of patients respectively. Asthma was third common comorbidity in $15(4.5 \%)$ patients. Other least commonly found comorbidity was thyroid disorders and CKD in $11(3.3 \%)$ patients.

Table 1 shows number of patients according to type of surgeries (as per NRC classification). In our study, clean wound surgeries were the most commonly performed surgeries in $34 \%$ patients followed by dirty wound surgeries in $31 \%$ of patients. Clean contaminated wound surgeries were third most commonly performed surgeries (27\%) during our study period. Contaminated wound surgeries were least commonly performed surgeries $(8 \%)$ in our study.

Table 1: Percentage of patient according to type of surgery (as per NRC classification).

\begin{tabular}{|ll|}
\hline Type of surgery & No. of patients (\%) \\
\hline Clean (class I) & $113(34)$ \\
\hline Clean contaminated (class II) & $90(27)$ \\
\hline Contaminated (class III) & $25(8)$ \\
\hline Dirty (class IV) & $102(31)$ \\
\hline
\end{tabular}

Table 2 shows that $\mathrm{p}$ value for the comparison among all 4 groups was $<0.001$ by Kruskal Wallis test followed by
Dunn's posthoc test for multiple comparison. Total duration of antimicrobial therapy during hospital stay among different surgical classes was significantly longer $(\mathrm{p}<0.001)$ in class II, class III, class IV as compared to class I. Also, the total duration of antimicrobials was longer $(p<0.001)$ in class III compare to class II and class IV compare to class III.

Table 2: Comparison of duration of antimicrobial therapy between different types of surgery.

\begin{tabular}{|lllll|} 
Class & Class & Class & $\begin{array}{l}\text { Class } \\
\text { III }\end{array}$ & $\begin{array}{l}\text { Class } \\
\text { IV }\end{array}$ \\
\hline Med-ian & 5 & 7 & 9 & 11 \\
\hline IQR & $4-5.5$ & $5-9$ & $9-11$ & $9-14$ \\
\hline Mean \pm SD & $4.94 \pm$ & $7 \pm$ & $9.68 \pm$ & $11.58 \pm$ \\
Range & 1.39 & 1.55 & 1.88 & 3.21 \\
\hline
\end{tabular}

Table 2.1: Comparison of $p$ value of duration of antimicrobial therapy between different types of surgery.

\begin{tabular}{|lc|}
\hline Comparison & P value \\
\hline Class I vs class II, III, IV & $<0.001$ \\
\hline Class II vs class III & $<0.001$ \\
\hline Class III vs class IV & $<0.001$ \\
\hline
\end{tabular}

$\mathrm{P}$ value for the comparison among all 4 groups was $<0.001$ by Kruskal Wallis test followed by Dunn's posthoc test for multiple comparison

Table 3 shows group wise distribution of antimicrobials. Nitroimidazole were the most commonly prescribed antimicrobials in $237(72 \%)$ of the patients. In nitromidazole group, metronidazole is only antimicrobial prescribed in $237(72 \%)$ of the patients.

Table 3: Percentage of various group of antimicrobials prescribed.

\begin{tabular}{|c|c|c|c|}
\hline Group of antimicrobials & $\begin{array}{l}\text { No. of patients } \\
(\mathrm{N}=330)(\%)\end{array}$ & Antimicrobials & $\begin{array}{l}\text { No. of } \\
\text { patients }(\%)\end{array}$ \\
\hline Nitromidazole (metronidazole) & $237(72)$ & Metronidazole & $237(72)$ \\
\hline \multirow{2}{*}{$\begin{array}{l}\text { Penicillins (amoxicillin + clavulanic acid, } \\
\text { piperacillin + tazobactum) }\end{array}$} & \multirow{2}{*}{$191(58)$} & Amoxicillin+clavulanic acid & $169(51.21)$ \\
\hline & & Piperacillin+tazobactum & $22(6.67)$ \\
\hline \multirow{4}{*}{$\begin{array}{l}\text { Cephalosporins (ceftriaxone, cefotaxime, } \\
\text { cefoperazone + sulbactum, cefazolin) }\end{array}$} & \multirow{4}{*}{$139(42)$} & Ceftriaxone & $69(20.90)$ \\
\hline & & Cefotaxim & $47(14.24)$ \\
\hline & & Cefoperazone+sulbactum & $13(3.93)$ \\
\hline & & Cefazolin & $10(3)$ \\
\hline Aminoglycoside (amikacin) & $55(17)$ & Amikacin & $55(16.67)$ \\
\hline \multirow{2}{*}{ Quinolones (ciprofloxacin, levofloxacin) } & \multirow{2}{*}{$49(15)$} & Ciprofloxacin & $3(10.60)$ \\
\hline & & Levofloxacin & $14(4.24)$ \\
\hline \multirow{2}{*}{ Carbapenems (imipenem, meropenem) } & \multirow{2}{*}{$14(4)$} & Imipenem & $9(2.72)$ \\
\hline & & Meropenem & $5(1.51)$ \\
\hline \multirow{4}{*}{$\begin{array}{l}\text { Others (antihelminthic, oxazolidinone, } \\
\text { macrolides, antifungal) }\end{array}$} & $22(6)$ & Antihelminthic (albendazole) & $9(2.72)$ \\
\hline & & Oxazolidinone (linezolid) & $9(2.72)$ \\
\hline & & Macrolides (azithromycin) & $2(0.6)$ \\
\hline & & Antifungal (miconazole) & $2(0.6)$ \\
\hline
\end{tabular}


Aminoglycosides were given in 55(17\%), quinolones in $49(15 \%)$ patients, carbapenems in $14(4 \%)$ patients and others groups like antihelminthic, oxazolidinone, macrolides, antifungal etc were prescribed in $22(6 \%)$ patients.

Figure 1 shows percentage of number of antimicrobials used per patient. Prescription containing single antimicrobials were $66(20 \%)$, two antimicrobials were 154 (47\%), three were 83 (25\%), and four were $18(5.5 \%)$. The most commomly prescibed single antimicrobial was amoxicillin and clavulinic acid in $53(16 \%)$ patients and most commonly prescribed two antimicrobials were metronidazole with amoxicillin and clavulinic acid in 46 (14\%) patients. Most frequently prescribed three antimicobials combination were metronidazole with cefotaxim and amikacin in 19 (6\%) patients. Most frequently prescribed four antimicobials combination were metronidazole, piperacillin and tazobactum, amikacin and cefixime in $9(3 \%)$ patients.

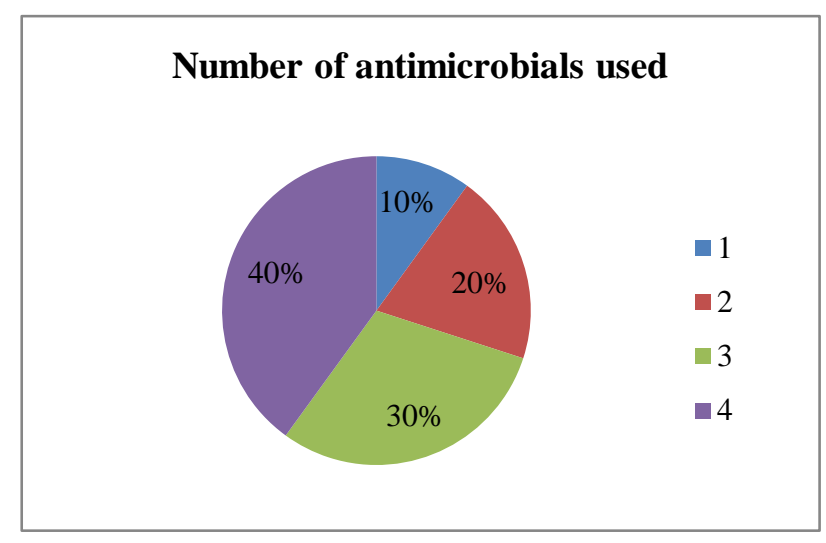

Figure 1 : Percentage of number of antimicrobials used per patient.

Table 4: Comparision of number of antimicrobials used per patient between different surgical classes.

\begin{tabular}{|lllll|} 
Class & Class I & $\begin{array}{l}\text { Class II } \\
\mathbf{N}=\mathbf{1 1 3}\end{array}$ & $\begin{array}{l}\text { Class III } \\
\mathbf{N}=90\end{array}$ & $\begin{array}{l}\text { Class IV } \\
\mathbf{N}=\mathbf{2 5}\end{array}$ \\
$\mathbf{N = 1 0 2}$ \\
\hline Median & 2 & 2 & 2 & 2 \\
\hline IQR & $1-2.5$ & $2-3$ & $2-3$ & $2-3$ \\
\hline Mean \pm & $1.90 \pm$ & $2.42 \pm$ & $2.2 \pm$ & $2.38 \pm$ \\
SD & 0.81 & 0.86 & 0.82 & 0.82 \\
\hline Range & $1-4$ & $1-4$ & $1-4$ & $1-4$ \\
\hline
\end{tabular}

Table 4.1: Comparison of $p$ value of number of antimicrobials used per patient between different surgical classes.

\begin{tabular}{|lc|}
\hline Comparison & P value \\
\hline Class I vs class II & $<0.001$ \\
\hline Class I vs class IV & $<0.001$ \\
\hline $\begin{array}{l}\text { P value for comparison among all 4, groups was }<0.001 \text { by } \\
\text { Kruskal Wallis test followed by Dunn's posthoc test for multiple } \\
\text { comparison }\end{array}$
\end{tabular}

Table 4 shows that p-value for comparison among all 4 groups was $<0.001$ by Kruskal Wallis test followed by Dunn's posthoc test for multiple comparison. The number of antibiotic prescribed per patient was significantly less $(\mathrm{p}<0.001)$ in class I as compare to class II and class IV surgeries.

133 antimicrobials (18\%) were prescribed with generic names and 598 antimicrobials (82\%) were prescribed with brand names. Out of 731 antimicrobial prescribed, intravenous route was administer in $683(93.5 \%)$, oral in $46(6.3 \%)$ and topical in 2 antimicrobials. Out of total, $29 \%$ antimicrobials were FDC. Most commonly prescribed FDC was Augmentin (amoxicillin and clavulanic acid), $18 \%$ of total antimicrobials. Second most commonly prescribed FDC was Piptaz (piperacillin and tazobactam), 9\% of total antimicrobials. Cefo SB (cefoperazone and sulbactam) was least commonly prescribed FDC, $2 \%$ of total antimicrobials.

Antimicrobial therapy was changed in 41(12.42\%) out of 330 patients after culture and sensitivity report. Most common change of antimicrobial therapy was penicillins to cephalosporins (21 of 41 patients) (eg-amoxicillin and clavulanic acid to cefixime). 263(36\%) antimicrobials were prescribed from hospital pharmacy whereas $468(64 \%)$ prescribed from outside pharmacy source.

Proton pump inhibitors (pantoprazole), NSAIDS (paracetamol, diclofenac etc) and antiemetics (ondansetron) were most commonly prescribed concomitant medications in $98 \%, 96 \%$ and $79 \%$ patients respectively. Nutritional supplements (multivitamins, calcium, vitamin C etc) were given in $47 \%$ patients. Synthetic enzymes (trypsin, chymotrypsin) were prescribed in $23 \%$ patients. Opioid analgesics (tramadol) were given in $15.7 \%$ patients. Patients having comorbidities like hypertension, asthma, diabetic mellitus were prescribed antihypertensive (atenolol, amlodipine, telmisartan etc) in $11 \%$ patients, antiasthamatics (salbutamol, ipratropium $\mathrm{Br}$ ) in $4.2 \%$ patients and antidiabetics medications like insulin preparations, oral hypoglycaemic in $11.5 \%$ and $6.3 \%$ patients respectively.

\section{DISCUSSION}

The present study was undertaken to review the prescribing trends of antimicrobials agents used post-operatively after surgical procedures.

We classified the patients according to type of surgery as per NRC classification as clean (class I), cleancontaminated (class II), contaminated (class III) and dirty (class IV) wound surgeries. In our study clean 113(34\%), dirty $102(31 \%)$ and clean contaminated 90(27\%) wound surgeries were most commonly performed surgeries (Table 1). Study done by Chawda et al found that dirty $(56.87 \%)$ and contaminated $(36.69 \%)$ wound surgeries were most commonly performed surgeries. ${ }^{8}$ 
Mean duration of antimicrobial therapy in our study was $7.92 \pm 3.52$ days. We found that total duration of antimicrobial therapy during hospital stay among different surgical classes was significantly longer $(p<0.001)$ in clean-contaminated (median-7), contaminated (median-9), dirty (median-11) wound surgeries as compared to clean wound surgeries (median-5) (Table 2).This suggest that patients in clean-contaminated, contaminated and dirty wound surgeries need more duration of antimicrobial therapy as compare to clean wound surgeries. However, these figures is an underestimate of the actual duration of antimicrobials as many patients were discharged with further antimicrobials prescribed at discharge which were not recorded in our study. Study done by Chawda et al found that duration of antimicrobial therapy was longer $(\mathrm{p}<0.001)$ in contaminated (median-12) and dirty wound surgeries (median-10) as compare to clean wound surgeries (median-8.5). ${ }^{8}$

With respect to the prescribing frequency of different antimicrobials groups, nitroimidazoles were the most commonly prescribed group in $237(72 \%)$ patients. The second most common were penicillins in $191(58 \%)$ patients, followed by cephalosporins group in 139(42\%) patients. Aminoglycosides were the fourth common in $55(17 \%)$ patients and quinolones were fifth common in $49(15 \%)$ patients (Table 3 ). The predominance of nitroimidazoles, penicillins and cephalosporins group was expected because these antimicrobials are recommended by most of the published guidelines for treatment and prophylaxis of infections occurring during or after various surgical procedures. ${ }^{9,10}$ These findings were similar to study done by Chawda et al and Kamath et al in which nitroimidazole (metronidazole) was most commonly given antimicrobial in $90.55 \%$ and $72 \%$ of subjects respectively. ${ }^{8,11}$

The average number of antimicrobials prescribed per prescription was $2.21 \pm 0.86$ with maximum of four antimicrobials per prescription. Use of single antimicrobial agent was seen in $20 \%$ of patients, two antimicrobials in $47 \%$ of patient followed by three antimicrobials (25\%). Four antimicrobials were prescribed in $8 \%$ of patients. This shows trend towards polypharmacy (Figure 1). The use of nitroimidazole with penicillins or cephalosporins was most frequently used combination in two drug regimen, this was followed by addition of aminoglycoside and/or quinolones in three and/or four drug regimens. This finding was similar to the study done by Kamath GS et al in which combination therapy of two antimicrobials $(57 \%)$ were most common preferred regimen followed by three $(30 \%){ }^{11}$ The reason for combining two antimicrobials in all studies could be to cover all aerobic and anerobic microorganisms responsible for surgical site infections.

We also compared the number of antimicrobials used per patients between different surgical classes. The number of antimicrobials prescribed per patient was significantly less $(\mathrm{p}<0.001)$ in clean wound surgeries as compare to clean- contaminated and dirty wound surgeries (Table 4). The reason being antimicrobials were prescribed in more number and for prolonged duration as infection is more and severe in other classes compared to clean wound surgeries. Similar findings were seen in study done by Chawda et al in which number of antimicrobials were significantly $(\mathrm{p}<0.05)$ more in number as compare to other surgical classes. ${ }^{8}$

In our study, out of total 731 antimicrobials, most of antimicrobials were prescribed with brands names 598 $(82 \%)$. Only $133(18 \%)$ antimicrobials were prescribed by generic names. Similar results were found in other studies done by Sharma et al (81.98\%) and Kamath et al (84\%) in which most of antimicrobials were prescribed by brand names. This could possibly because of non-availability of antimicrobials in hospital pharmacy. ${ }^{11,13}$

In our study, intravenous route was most common route of antimicrobials administration $(93.5 \%$ of all antimicrobials). This was expected because bowel movements require some time to become normal after performing many surgeries. Hence, it is convenient to use intravenous route of administration in these patients. This was similar to study done by Brethis CS et al in which most common route of administration of antimicrobials was intravenous $(61 \%){ }^{12}$

In our study, out of total antimicrobials prescribed to the patients, $29 \%$ antimicrobials were fixed dose combination (FDC). Most commonly prescribed FDC was amoxicillin and clavulanic acid (18\%) followed by piperacillin and tazobactam) (9\%) of total antimicrobials (Figure 6). This was possibly due to they cover gram positive and gram negative bacteria responsible for surgical site infections and they are cheaper as compare to other FDCs. However, study done by Sharma $\mathrm{P}$ et al found that piperacillin and tazobactam was common FDC used (20.5\%), followed by ceftriaxone and sulbactam $(13 \%){ }^{14}$

In our study, antimicrobial therapy was changed in $41(12.42 \%)$ out of 330 patients after culture and sensitivity report. Most common change of antimicrobial therapy was penicillins to cephalosporins (21 of 41 patients) (eg.amoxicillin and clavulanic acid to cefixime). This shows that, in culture and sensitivity report microorganisms responsible for SSI were sensitive to cephalosporins.

In our study $64 \%$ drugs were prescribed from outside pharmacy. The reason was either these drugs were not on hospital schedule list or due to scarcity in the stocks of scheduled drugs. Some most commonly prescribed drugs which were not on hospital schedule included cefixime, ciprofloxacin and doxycycline. Hence, we recommend our hospital pharmacy to include these drugs in hospital schedule list. Also, sometimes drugs like ceftriaxone, amikacin, amoxicillin and clavulanic acid, piperacillin and tazobactum, cefoperazone and sulbatctam were not available in hospital pharmacy. We also recommend hospital pharmacy to take necessary measures to assure 
continuous availability of these commonly used antimicrobials at all times.

The major limitation of our study was the rate of surgical site infection in different types of surgeries which was not evaluated. Our study did not include the antimicrobials prescribed at discharge so actual total duration of antimicrobial use will be more than what is reported in our study.

We also studied prescription of drugs from hospital or outside pharmacy. Hence, we can give corrective recommendations to the hospital pharmacy. Efforts to prescribe available best choice of drug from hospital schedule will help in reducing the cost of therapy. Further studies can be done to assess the effectiveness of current prescribing pattern and influence on rate of surgical site infections.

\section{CONCLUSION}

The prescription pattern shows that nitroimidazoles were the most commonly prescribed group of antimicrobials in $72 \%$ of patients followed by penicillins $(58 \%)$ and cephalosporins (42\%). Total duration of antimicrobial therapy, number of anti-microbial used was more in cleancontaminated, contaminated, dirty wound surgeries as compare to clean wound surgeries. Our study provides a framework for continuous prescription audit of antimicrobials in a hospital setting and thus can help in rational use of antimicrobials in post-operative surgical patients.

\section{ACKNOWLEDGEMENTS}

Authors are specially thankful to Dr. S. Dharap sir, Professor and Head of Department of Surgery for giving permission to collect the data from surgery wards.

Funding: No funding sources

Conflict of interest: None declared

Ethical approval: The study was approved by the Institutional Ethics Committee

\section{REFERENCES}

1. Bulander RE, Dunn DL, Beilman GJ. Surgical Infections. In: Brunicardi F, Andersen DK, Billiar TR, Dunn DL, Kao LS, Hunter JG, Matthews JB, eds. Schwartz Principles of Surgery. 11th ed. USA: McGraw-Hill; 2019: 157-182.

2. Lamont P. Surgical infection. In: Williams NS, Andrew W, McCaskie, eds. Bailey and Love Short Practice of Surgery. 27th ed. London: Hodder Arnold; 2018: 42-56.
3. Lampiris HW, Maddix DS. Clinical Use of Antimicrobial Agents. In: Katzung BG, Susan B, Trevor AJ, eds. Basics and clinical pharmacology. 11th ed. USA: McGraw-Hill; 2009:895- 898.

4. World Health Organization. Introduction to drug utilization research. National Institute of Food and Drug Safety Evaluation in Republic of Korea; 2003.

5. William AP. Penicillins, Cephalosporins, and Other $\beta$ Lactam Antibiotics. In: Brunton LL, Chabner BA, Knollmann BC, eds. Goodman \& Gilman's Pharmacological Basis of therapeutics. 13th edn. USA: McGraw-Hill; 2010: 1477-1503.

6. Kulshretha S, Aggrawal KK. Survey of pattern of antimicrobial use in teaching hospital. Indian $\mathbf{J}$ Pharmacol. 2010;16(1):395.

7. Creative Research Systems. Sample Size Calculator, 1982. Available at: https://www.surveysystem.com/ sscalc.htm. Accessed on 7 March 2021.

8. Chawda HM, Patel M, Mandaviya D, Barvaliya M, Patel T, Tripathi DC. Utilization pattern of antimicrobials among patients undergone midline laparotomy in four tertiary care teaching hospitals of Gujarat, India: multi-centric retrospective study. Asian J Pharm Clin Res. 2013;6(5):145-50.

9. Allen G, Fapic C. New CDC guideline for the prevention of surgical site infections. Am Nurse Today. 2017;12(8):6-10.

10. National Centre for Disease Control. National treatment guidelines for antimicrobial use in infectious diseases. 2016: 1-64.

11. Kamath SG, Varun HV, Rani UD, Aithal S, Patil UN. Prescribing patterns of antimicrobials in surgical departments in a tertiary care hospital in South India. Orthopaedics. 2014;44:28-57.

12. Brethis CS, Thamizharasan S, Sridevi SA, Kalaiselvi B, Singh MB, Vijaykumar K. A prospective study of the pattern of use of antimicrobial agents in surgical prophylaxis in a tertiary care centre. Int $\mathbf{J}$ Pharm Pharm Sci. 2017;9(1):283-7.

13. Sharma N, Bhargava M, Mahawar D, Parakh R. Usage of antibiotics in postoperative patients in a tertiary care teaching hospital in India. Internat $\mathbf{J}$ Pharm Res Bio-Sci. 2014;3(2):99-105.

14. Sharma P, Goel D. Utilization Assessment of antimicrobial prophylaxis in surgical patients at tertiary care hospital. Saudi J Health Sci. 2018;7:23-7.

Cite this article as: Hotwani JH, Madkholkar NH. Study of prescription pattern of antimicrobials used in post-operative surgical patients in tertiary care hospital. Int J Basic Clin Pharmacol 2021;10:627-32. 